\title{
Electrodeposition of Co and Co-Fe Films on Platinum and on Copper Substrates
}

\author{
M. C. Vilchenski", G. C. Silva, A. V. Benedetti and P. T. A. Sumodjo ${ }^{\text {a) }}$ \\ Instituto de Química da Universidade Estadual Paulista, UNESP. Departamento de Físico- \\ Química, CP. 355, 14801-970 Araraquara, SP, Brasil.

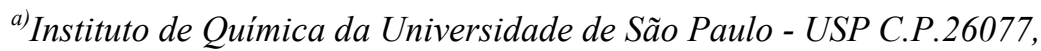 \\ 05599 São Paulo SP, Brasil.
}

Received 14 September 2002; accepted in revised form 30 October 2002

\begin{abstract}
CoFe films were electrodeposited on platinum and copper from an acidic sulfate bath. The deposits surface morphology was analyzed using SEM and composition was determined by XPS. For deposition potentials lower than $-0.80 \mathrm{~V}(\mathrm{SCE})$, the anodic stripping voltammogram showed a complex anodic current peak evidencing the complexity of the deposition process. Analysis of the deposition current transient curves showed that the nature of the substrate influenced the kinetic of the process: it was observed an instantaneous nucleation on $\mathrm{Pt}$ and a progressive nucleation on $\mathrm{Cu}$.

Pure cobalt and cobalt-iron films formed on $\mathrm{Pt}$ and on $\mathrm{Cu}$ presented binding energies for $\mathrm{Co} 2 \mathrm{p}_{3 / 2}$ signal corresponding to cobalt oxide, while the $\mathrm{Fe} 2 \mathrm{p}_{3 / 2}$ signal is related to metallic iron. The corresponding metallic cobalt was observed for the Co2 $\mathrm{p}_{3 / 2}$ signal with binding energy of about $778.0 \mathrm{eV}$.
\end{abstract}

Keywords: electrodeposition, $\mathrm{Co}$ and $\mathrm{Co}+\mathrm{Fe}$ films, platinum, copper, cyclic voltammetry, chronoamperometry, SEM, XPS.

\section{Introdução}

Filmes e ligas depositados eletroquimicamente e particularmente em multicamadas têm interesse tecnológico porque apresentam a propriedade de magnetoresistência gigante [1,2]. Muitos destes materiais são preparados por

\footnotetext{
* Corresponding author. E-mail address: marcvilc@posgrad.iq.unesp.br
} 
sputtering, evaporação, deposição por vapor químico (CVD) e outros processos de deposição à vácuo. No entanto, a eletrodeposição tem várias vantagens sobre esses métodos [3], tais como, os equipamentos são relativamente simples e baratos, dentre outras. Comparada com os processos de deposição à vácuo, onde os substratos são aquecidos bem acima da temperatura ambiente, a eletrodeposição normalmente é feita a temperatura ambiente. Além disso, composição, morfologia e propriedades magnéticas dos depósitos podem ser ajustadas através do controle de parâmetros como potencial, corrente, $\mathrm{pH}$ e composição do banho. A eletrodeposição de filmes de cobalto é particularmente interessante porque estudos prévios indicaram que multicamadas de $\mathrm{Co} / \mathrm{Cu}$, $\mathrm{CoNiCu} / \mathrm{Cu}, \mathrm{Co} / \mathrm{Pt}, \mathrm{Fe} / \mathrm{Cu}, \mathrm{Fe}_{80} \mathrm{Ni}_{20} / \mathrm{Cu}$ são materiais extremamente promissores e podem ter um enorme efeito de magnetoresistência gigante (MRG) [2,4,5]. Esses filmes e ligas têm uma ampla aplicação em indústrias eletrônicas, de computadores, e de fitas de gravação magnética [6].

A maioria dos estudos de eletrodeposição encontrados na literatura trata da morfologia dos depósitos relacionando-a com as condições de eletrodeposição. Esses estudos, apesar de fundamentais para o desenvolvimento de novos materiais, não são enfocados na elucidação da cinética ou mecanismo das reações de eletrodeposição, especialmente para a deposição de ligas [7]. Nota-se também que um dos substratos mais utilizados é o de carbono vítreo, para minimizar a influência da reação de geração de hidrogênio. Não obstante, é bem conhecido que a natureza do substrato é um fator muito importante para determinar não apenas a morfologia do depósito mas também a cinética dos processos de eletrocristalização [8]. Além disso, usualmente os filmes são depositados sobre silício ou cobre.

Apesar dos múltiplos aspectos considerados nestes estudos, o mecanismo de nucleação, os processos de cristalização, e principalmente a natureza da codeposição anômala envolvidos na eletrodeposição de filmes e ligas de cobaltoferro têm recebido pouca atenção, comparados com a grande quantidade de estudos da eletrodeposição de níquel e cobre [9-11]. 
Neste trabalho são discutidos resultados da eletrodeposição de filmes de Co-Fe sobre substratos de platina e de cobre e sua caracterização morfológica e composicional através de microscopia eletrônica de varredura e espectroscopia fotoeletrônica de Raios-X, respectivamente.

\section{Experimental}

Os estudos eletroquímicos com eletrodos de disco foram realizados em uma célula eletroquímica convencional. Para os ensaios onde os eletrodos utilizados eram placas, foi utilizada uma célula horizontal EG\&G parc ("flat cell") com capacidade para $250 \mathrm{~mL}$. Os eletrodos de trabalho foram discos $\left(3.14 \mathrm{~mm}^{2}\right) \mathrm{e}$ placas $\left(0.196 \mathrm{~cm}^{2}\right)$ de platina e cobre. Um fio de platina e um eletrodo de calomelanos saturado (ECS) foram usados como eletrodos auxiliar e de referência, respectivamente. Todos os potenciais são referidos ao ECS. O banho usado para as eletrodeposições consistiu de $\mathrm{Na}_{2} \mathrm{SO}_{4} 0.5 \mathrm{M}+\mathrm{H}_{3} \mathrm{BO}_{3} 0.4 \mathrm{M}$ com pH 3, contendo $\mathrm{CoSO}_{4} 0.2 \mathrm{M}$ ou $\mathrm{CoSO}_{4} 0.2 \mathrm{M}+\mathrm{FeSO}_{4}(5,10$ e $20 \mathrm{mM})$. A eletrodissolução dos depósitos foi realizada no eletrólito suporte: $\mathrm{Na}_{2} \mathrm{SO}_{4} 0.5 \mathrm{M}$ $+\mathrm{H}_{3} \mathrm{BO}_{3} 0.4 \mathrm{M}$ com $\mathrm{pH}$ 3. Todos os ensaios foram realizados a $25{ }^{\circ} \mathrm{C}$. Como técnicas utilizou-se a voltametria, salto de potencial, SEM, EDX, e XPS. As medidas de XPS foram realizadas irradiando as amostras com Raios-X monoenergéticos $(\mathrm{Mg} \mathrm{K \alpha} 1253.6 \mathrm{eV})$. O espectro foi registrado com e sem bombardeamento da superfície com íons $\mathrm{Ar}^{+}$(sputter). Todos os valores de energia foram calibrados com o valor do carbono $(\mathrm{C}(1 \mathrm{~s})=284.8 \mathrm{eV})[12]$.

\section{Resultados e Discussão}

A Fig. 1a mostra os voltamogramas cíclicos do substrato de platina em solução de sulfato de cobalto $0.2 \mathrm{~mol} \mathrm{~L}^{-1}+$ ácido bórico $0.4 \mathrm{~mol} \mathrm{~L}^{-1}+0.5 \mathrm{~mol} \mathrm{~L}^{-1} \mathrm{de}$ sulfato de sódio + sulfato ferroso $\left[(0,0.5,1\right.$ e 2$\left.) \times 10^{-3} \mathrm{~mol} \mathrm{~L}^{-1}\right], \mathrm{pH} 3, \omega=0 \mathrm{rpm}$, e $\mathrm{v}=5 \mathrm{mV} \mathrm{s}^{-1}$. Na região de redução, em solução de cobalto puro, observa-se dois picos de corrente em $-0.86 \mathrm{~V}$ e $-1.00 \mathrm{~V}$ em baixas velocidades de varredura $\left(\mathrm{v}=5\right.$ e $\left.10 \mathrm{mV} \mathrm{s}^{-1}\right)$. Para $\mathrm{v} \geq 20 \mathrm{mV} \mathrm{s}^{-1}$ é observado apenas um pico de redução, cujo potencial desloca-se com o aumento de v. Este aumento da correspondente 
corrente catódica pode estar relacionado ao aumento da densidade de núcleos e crescimento de cristais [13]. Em potenciais mais negativos $(\mathrm{E}<-1.4 \mathrm{~V})$, o aumento na corrente catódica está associado a reação de evolução de hidrogênio (REH) [13]. No sentido de potenciais crescentes (varredura anódica), os voltamogramas apresentam um pico largo de corrente (principal), cujo potencial desloca-se com o aumento de $\mathrm{v}$. Para $\mathrm{v}=5 \mathrm{mV} \mathrm{s}^{-1}$, observa-se um pequeno prépico de oxidação em $-0.40 \mathrm{~V}$. Este pré-pico não é observado para $\mathrm{v}>5 \mathrm{mV} \mathrm{s}^{-1}$. Para $\mathrm{v} \geq 20 \mathrm{mV} \mathrm{s}^{-1}$, um pequeno ombro de corrente aparece após o pico principal. A corrente deste ombro também aumenta com o aumento de v. Os voltamogramas obtidos com a presença de ferro em solução apresentam no sentido decrescente de potenciais (varredura catódica) apenas um pico de corrente catódica. Tal pico corresponde à codeposição de ambos os metais Co e Fe a partir da redução de $\mathrm{Co}^{2+}$ para $\mathrm{Co}^{0}$ e $\mathrm{Fe}^{2+}$ para $\mathrm{Fe}^{0}$. A observação de um único pico de corrente catódico para a redução de ambos os íons $\mathrm{Co}^{2+}$ e $\mathrm{Fe}^{2+}$ aparece como uma característica da eletrodeposição destas ligas [14]. Esta característica pode estar relacionada ao fenômeno da codeposição anômala. No sentido crescente de potenciais (varredura anódica) observa-se, para $\mathrm{v}=5 \mathrm{mV} \mathrm{s}^{-1}$, um pico largo duplo de corrente anódica. Para as demais velocidades este pico largo aparece ora precedido ora sucedido por um ombro.

A Figura 1b mostra os voltamogramas cíclicos do substrato de cobre em solução de sulfato de cobalto $0.2 \mathrm{~mol} \mathrm{~L}^{-1}+$ ácido bórico $0.4 \mathrm{~mol} \mathrm{~L}^{-1}+0.5 \mathrm{~mol} \mathrm{~L}^{-1} \mathrm{de}$ sulfato de sódio + sulfato ferroso $\left[(0,0.5,1\right.$ e 2$\left.) \times 10^{-3} \mathrm{~mol} \mathrm{~L}^{-1}\right], \mathrm{pH} 3, \omega=0 \mathrm{rpm}$, $\mathrm{e} \mathrm{v}=5 \mathrm{mV} \mathrm{s}^{-1}$. Na varredura catódica os voltamogramas em solução de cobalto e cobalto + ferro $\left(5,10\right.$ e $\left.2 \times 10^{-2} \mathrm{~mol} \mathrm{~L}^{-1}\right)$ apresentam um único pico. Este pico desloca seu valor de potencial para valores mais negativos à medida que a concentração de ferro aumenta. Este deslocamento também foi observado para o substrato de platina. Para os aumentos de corrente observados nos voltamogramas do substrato de cobre no sentido negativo de potenciais (varredura catódica) valem as mesmas observações feitas para o substrato de platina. No sentido crescente de potenciais (varredura anódica), os voltamogramas apresentam um pico largo de corrente (principal) cujo potencial 
desloca-se para valores mais positivos com o aumento da concentração de ferro. Este pico aparece sucedido por um ombro de corrente em aproximadamente 0.1 $\mathrm{V}$ apenas para o voltamograma obtido em solução com concentração de ferro igual $2 \times 10^{-2} \mathrm{~mol} \mathrm{~L}^{-1}$. Para $\mathrm{v}=1 \mathrm{mV} \mathrm{s}^{-1} \mathrm{o}$ pico principal de oxidação aparece desdobrado nos voltamogramas em todas as concentrações de ferro em solução, sugerindo a presença de mais de uma fase na liga ou de diferentes espécies sendo dissolvidas.
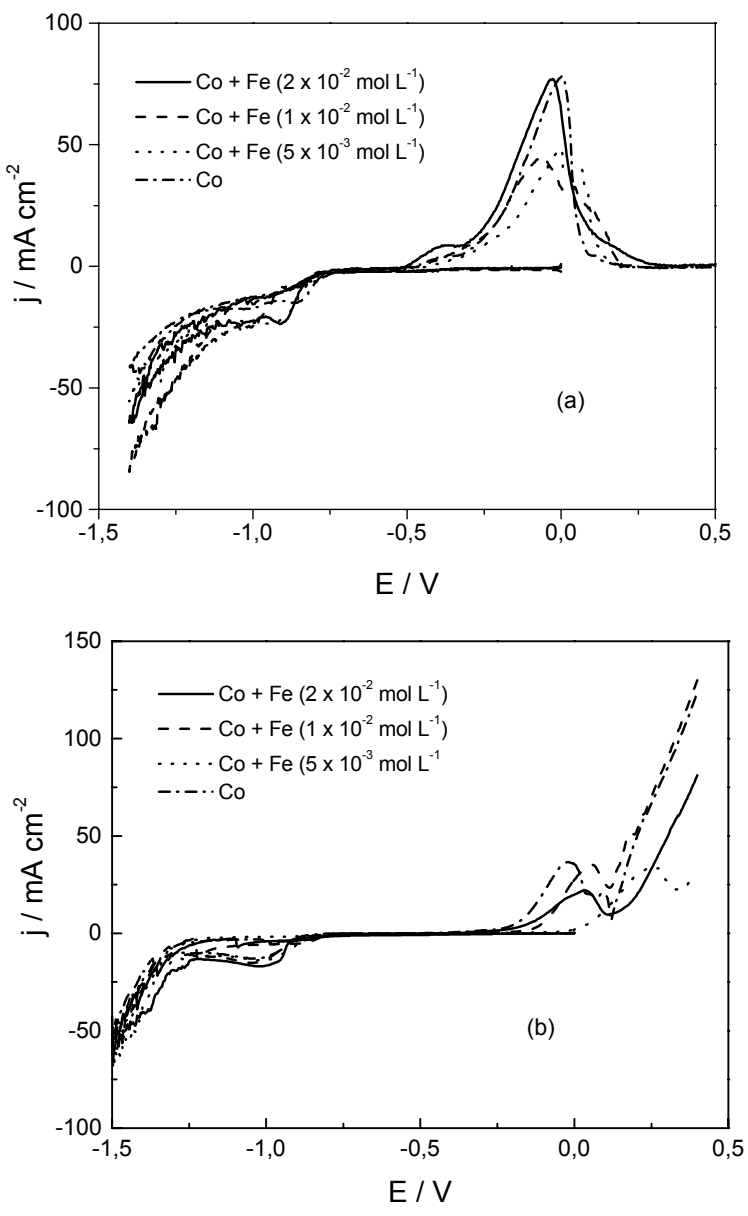

Figura 1. Voltamogramas cíclicos obtidos para os substratos de platina (a) e cobre (b) nas soluções de sulfato de cobalto $0.2 \mathrm{~mol} \mathrm{~L}^{-1}+$ ácido bórico $0.4 \mathrm{~mol}$ $\mathrm{L}^{-1}+0.5 \mathrm{~mol} \mathrm{~L}^{-1}$ de sulfato de sódio + sulfato ferroso $\left[(0,0.5 ; 1\right.$ e 2$) \times 10^{-2} \mathrm{~mol} \mathrm{~L}^{-}$ ${ }^{1}$ ], $\mathrm{pH} \mathrm{3, \omega}=0 \mathrm{rpm}, \mathrm{v}=5 \mathrm{mV} \mathrm{s}^{-1}$.

Para efeito de comparação, foi registrado o voltamograma cíclico do substrato de platina em solução de sulfato ferroso $2 \times 10^{-2} \mathrm{~mol} \mathrm{~L}^{-1}+$ ácido bórico $0.4 \mathrm{~mol} \mathrm{~L}^{-1}$ 
$+0.5 \mathrm{~mol} \mathrm{~L}^{-1}$ de sulfato de sódio para $\mathrm{v}=5 \mathrm{mV} \mathrm{s}^{-1}$. Verifica-se que o voltamograma apresenta no sentido decrescente de potenciais (varredura catódica) apenas um pico de corrente em $-1.09 \mathrm{~V}$ devido à redução de $\mathrm{Fe}^{2+}$ para $\mathrm{Fe}^{0}$. No sentido crescente de potenciais (varredura anódica) observa-se a presença de um ombro em $-0.47 \mathrm{~V}$ e um pico em $-0.29 \mathrm{~V}$ (Fig. 2a). Para o substrato de cobre também foi registrado o voltamograma cíclico em solução de sulfato ferroso $2 \times 10^{-2} \mathrm{~mol} \mathrm{~L}^{-1}+$ ácido bórico $0.4 \mathrm{~mol} \mathrm{~L}^{-1}+0.5 \mathrm{~mol} \mathrm{~L}^{-1}$ de sulfato de sódio para $\mathrm{v}=5 \mathrm{mV} \mathrm{s}^{-1}$. Observa-se que o voltamograma também apresenta, no sentido decrescente de potenciais, apenas um pico de corrente catódica em aproximadamente $-1.09 \mathrm{~V}$. No sentido crescente de potenciais (varredura anódica) observa-se a presença de apenas um pico em $-0.58 \mathrm{~V}$ (Fig. $2 b)$.
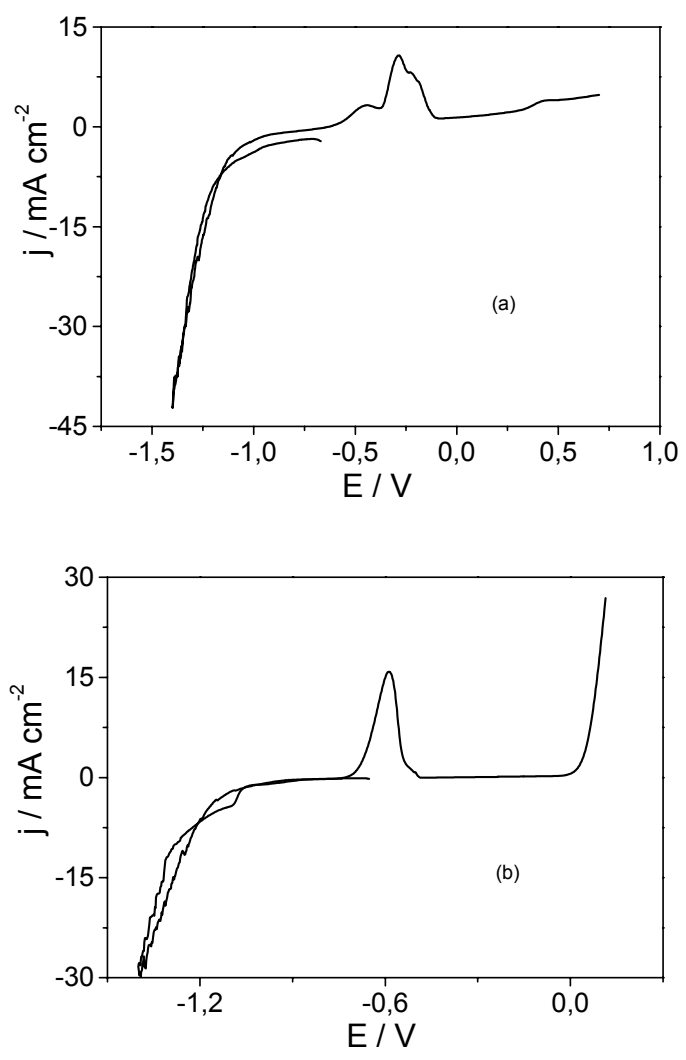

Figura 2. Voltamogramas cíclicos obtidos para o substrato de platina (a) e cobre (b) em solução de sulfato ferroso $2 \times 10^{-2} \mathrm{~mol} \mathrm{~L}^{-1}+$ ácido bórico $0.4 \mathrm{~mol} \mathrm{~L}^{-1}+0.5$ mol L ${ }^{-1}$ de sulfato de sódio, $\mathrm{pH} \mathrm{3, \omega}=0 \mathrm{rpm}, \mathrm{v}=5 \mathrm{mV} \mathrm{s}^{-1}$. 
Verifica-se que a dissolução do ferro em uma solução contendo apenas ferro sobre substrato de cobre apresenta apenas um pico, enquanto que sobre platina o pico é desdobrado. Isto sugere que o substrato de platina interage diferentemente do cobre com os metais Co e Fe e que provavelmente o potencial de oxidação das primeiras camadas do depósito é diferente do obtido para as demais camadas, ou existem fases da liga (grãos de composição diferente) que são oxidadas em potenciais diferentes, dependendo do substrato.

Foram realizados ensaios de dissolução do filme de cobalto em solução do eletrólito suporte (ácido bórico $0.4 \mathrm{~mol} \mathrm{~L}^{-1}+0.5 \mathrm{~mol} \mathrm{~L}^{-1}$ de sulfato de sódio, $\mathrm{pH}$ 3). Os filmes foram depositados potenciostaticamente com $E_{d}$ entre -0.60 e -1.25 V durante 1 minuto em solução contendo sulfato de cobalto $0.2 \mathrm{~mol} \mathrm{~L}^{-1}+$ ácido bórico $0.4 \mathrm{~mol} \mathrm{~L}^{-1}+0.5 \mathrm{~mol} \mathrm{~L}^{-1}$ de sulfato de sódio, $\mathrm{pH}$ 3. Os voltamogramas foram obtidos a $50 \mathrm{mV} \mathrm{s}^{-1}$, em solução de eletrólito isenta das espécies eletroativas.

A Fig. 3 mostra os voltamogramas do substrato de platina em solução quiescente de eletrólito suporte após deposição de cobalto em potencial constante em diferentes potenciais. Os voltamogramas apresentam um pico principal de dissolução que se desloca para potenciais mais positivos à medida que o potencial de deposição torna-se mais negativo. Este deslocamento deve-se ao aumento da espessura do filme que dificulta a remoção de todo o material, até por difusão para fora da superfície do eletrodo. Um pré-pico de oxidação é observado para potenciais de deposição $\mathrm{E}_{\mathrm{d}} \leq-0.90 \mathrm{~V}$. Verifica-se que para potenciais de deposição mais negativos que $-0.80 \mathrm{~V}$ o pico agudo de corrente que aparece nos voltamogramas de dissolução é atribuído à dissolução do cobalto $[15,16]$; já o pré-pico de oxidação pode estar associado a diferentes estados energéticos de cobalto, fases ou hidretos de cobalto.

Nos voltamogramas obtidos na presença de ferro em solução, nas três concentrações, observa-se que o pico principal de oxidação aparece desde os mais baixos potenciais de deposição (Fig. 4a). Isso indica que há deposição apreciável de ferro. Este pico, na presença de ferro em solução, passa a ser devido ao processo de dissolução simultânea dos metais para as menores 
concentrações de ferro. Para $E_{d}=-0.90 \mathrm{~V}$ (Figura 4b) nota-se que, no voltamograma de dissolução no eletrólito suporte o pico principal de oxidação é mais agudo que os voltamogramas cuja solução de deposição possuía ferro.

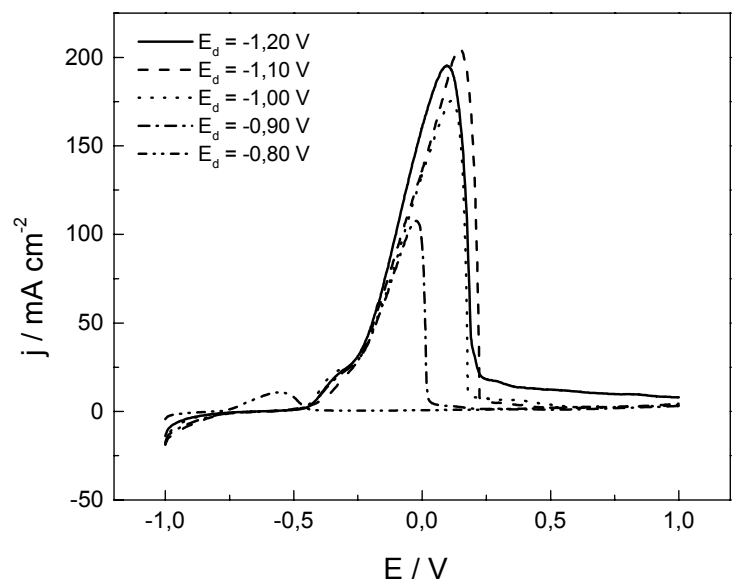

Figura 3. Voltamogramas cíclicos obtidos para o substrato de platina em solução do eletrólito suporte após deposição potenciostática na solução de sulfato de cobalto $0.2 \mathrm{~mol} \mathrm{~L}^{-1}+$ ácido bórico $0.4 \mathrm{~mol} \mathrm{~L}^{-1}+0.5 \mathrm{~mol} \mathrm{~L}^{-1}$ de sulfato de sódio, $\mathrm{pH} \mathrm{3, \omega}=0 \mathrm{rpm}, \mathrm{v}=5 \mathrm{mV} \mathrm{s}^{-1}$.

Pode-se observar também que em todos os voltamogramas, exceto para os obtidos em solução cuja concentração de ferro é de $2 \times 10^{-2} \mathrm{~mol} \mathrm{~L}^{-1}$, o pico principal é precedido por um ombro de corrente. Para esta concentração de ferro em solução $\left(2 \times 10^{-2} \mathrm{~mol} \mathrm{~L}^{-1}\right)$, os voltamogramas apresentam-se, neste $\mathrm{E}_{\mathrm{d}}(-0.90$ V), com um pico principal duplo. Pode-se perceber também que os voltamogramas registrados para todas as concentrações de ferro apresentam ombros de corrente após o pico principal entre 0.0 e $0.5 \mathrm{~V}$. Estes ombros apresentam uma corrente maior nos voltamogramas registrados em soluções contendo ferro. Voltamogramas obtidos em soluções contendo $5 \times 10^{-3} \mathrm{~mol} \mathrm{~L}^{-1}$ de ferro mostram estes ombros de corrente apenas em $E_{d} \leq-0.95 \mathrm{~V}$. Já os voltamogramas da solução com $1 \times 10^{-2} \mathrm{~mol} \mathrm{~L}^{-1}$ de ferro mostram estes ombros para $E_{d} \leq-1.00 \mathrm{~V}$, e para a maior concentração de ferro em solução $\left(2 \times 10^{-2}\right.$ mol $\mathrm{L}^{-1}$ ), os voltamogramas apresentam estes ombros praticamente em todos os potenciais de deposição. Isto pode sugerir a formação de alguma fase Co-Fe, que é facilitada com o aumento da concentração de Fe. 

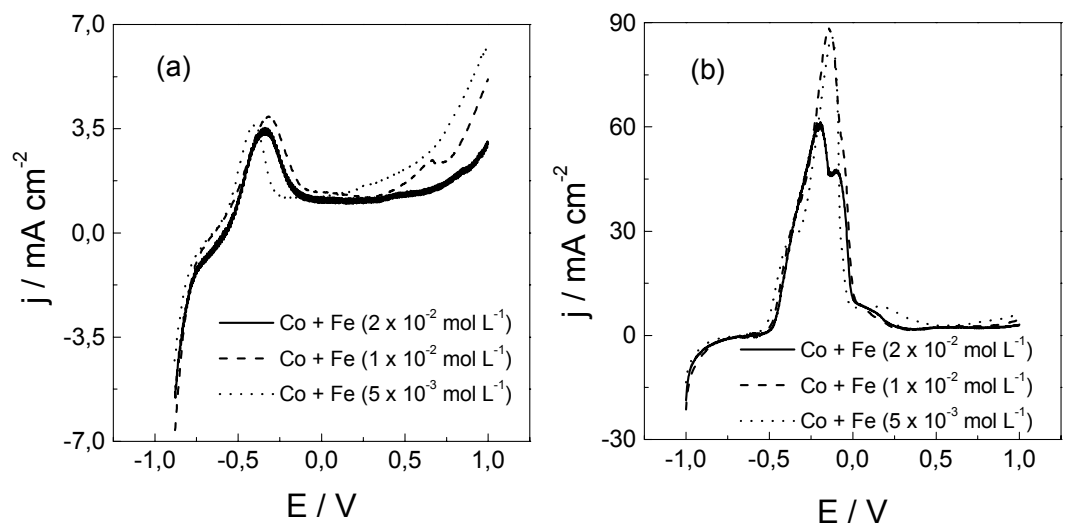

Figura 4. Voltamogramas cíclicos obtidos para o substrato de platina em solução do eletrólito suporte após deposição potenciostática na solução de sulfato de cobalto $0.2 \mathrm{~mol} \mathrm{~L}^{-1}+$ ácido bórico $0.4 \mathrm{~mol} \mathrm{~L}^{-1}+0.5 \mathrm{~mol} \mathrm{~L}^{-1}$ de sulfato de sódio + sulfato ferroso $\left[(0,0.5 ; 1 \mathrm{e} 2) \times 10^{-2} \mathrm{~mol} \mathrm{~L}^{-1}\right], \mathrm{pH} 3, \omega=0 \mathrm{rpm}, \mathrm{v}=5 \mathrm{mV} \mathrm{s}^{-1}$, (a) $\mathrm{E}_{\mathrm{d}}=-0.70 \mathrm{~V}$, (b) $\mathrm{E}_{\mathrm{d}}=-0.90 \mathrm{~V}$.

As Figs. 5a e 5b mostram as respostas I-t para os substratos de platina e cobre em solução de sulfato de cobalto $0.2 \mathrm{~mol} \mathrm{~L}^{-1}+$ ácido bórico $0.4 \mathrm{~mol} \mathrm{~L}^{-1}+0.5 \mathrm{~mol} \mathrm{~L}^{-1}$ de sulfato de sódio na ausência e na presença de sulfato ferroso $[(0,0.5 ; 1$ e 2)x $10^{-2} \mathrm{~mol} \mathrm{~L}^{-1}$ ], com $\omega=0 \mathrm{rpm}, \mathrm{E}_{\mathrm{d}}=-0.95 \mathrm{~V}$. De modo geral, as respostas I-t, obtidas sobre substrato de platina com soluções contendo ferro, mostram correntes mais intensas que a solução contendo apenas cobalto. Está claro por esta observação que a deposição do Co-Fe ocorre por um mecanismo complexo e provavelmente múltiplo conforme já apontado na literatura [17]. Em tempos curtos de deposição o decaimento de corrente do transiente corresponde à carga da dupla camada elétrica. Em seguida há um aumento de corrente devido ao crescimento de uma nova fase ou a um aumento do número de núcleos. Com o crescimento dos núcleos, a sobreposição das zonas de difusão vizinhas ocasiona um aumento da corrente até um máximo, que é seguido por uma porção de decaimento que se aproxima da corrente correspondente à difusão planar para toda superfície do substrato [18]. A forma dos transientes experimentais é típica de um processo de nucleação tridimensional controlado por difusão hemisférica para o crescimento dos cristalitos [19,20]. Sendo assim, um critério conveniente para distinguir dois casos extremos de cinética de nucleação é apresentar os 
dados experimentais em um gráfico adimensional; $\left(\mathrm{i} / \mathrm{i}_{\max }\right)^{2} \mathrm{vs} . \mathrm{t} / \mathrm{t}_{\max }$, resultando nas equações:

$$
\left(\frac{i}{i_{\max }}\right)^{2}=1,9542\left(\frac{t}{t_{\max }}\right)^{-1}\left\{1-\exp \left[-1,2564\left(\frac{t}{t_{\max }}\right)\right]\right\}^{2}
$$

para nucleação instantânea, e

$$
\left(\frac{i}{i_{\max }}\right)^{2}=1,2254\left(\frac{t}{t_{\max }}\right)^{-1}\left\{1-\exp \left[-2,3367\left(\frac{t}{t_{\max }}\right)\right]\right\}^{2}
$$

para nucleação progressiva.
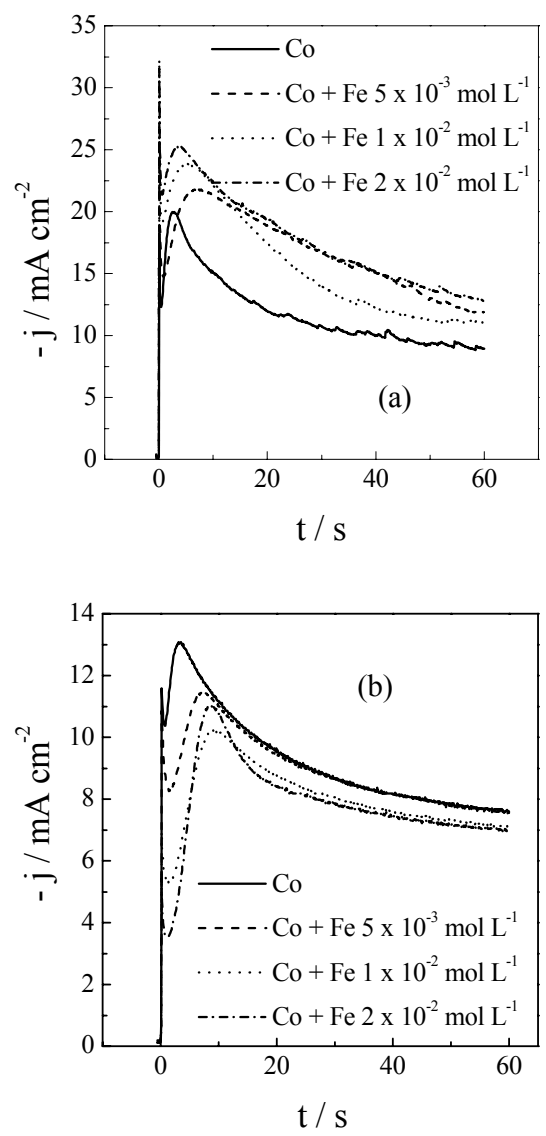

Figura 5. Sequência de transientes potenciostáticos de corrente, obtidos para os substratos de platina (a) e cobre (b), em solução de sulfato de cobalto $0.2 \mathrm{~mol} \mathrm{~L}^{-1}$ + ácido bórico $0.4 \mathrm{~mol} \mathrm{~L}{ }^{-1}+0.5 \mathrm{~mol} \mathrm{~L}{ }^{-1}$ de sulfato de sódio + sulfato ferroso [ $(0$, $0.5,1$ e 2) $\left.\left.\times 10^{-3} \mathrm{~mol} \mathrm{~L}^{-1}\right)\right], \mathrm{pH} 3, \omega=0 \mathrm{rpm}, \mathrm{E}_{\mathrm{d}}=-0.95 \mathrm{~V}, \mathrm{t}_{\mathrm{d}}=120 \mathrm{~s}$.

Os resultados dos transientes com o eletrodo de platina mostraram uma concordância razoável com o modelo de nucleação instantânea para potenciais de 
deposição menos negativos $(-0.85 \mathrm{e}-0.90 \mathrm{~V})$ no intervalo da relação $\mathrm{t} / \mathrm{t}_{\max }$, ou seja, até $\mathrm{t} / \mathrm{t}_{\max }$ em torno de 2 . Já sobre eletrodo de cobre ocorreu uma maior concordância no intervalo $\mathrm{t} / \mathrm{t}_{\max }$ em torno de 2 para o modelo de nucleação progressiva.

As micrografias eletrônicas de varredura mostraram que os filmes apresentam uma rugosidade maior quando depositados sobre eletrodos de cobre e o tamanho das partículas esféricas diminuem com o aumento da concentração de ferro na solução (Figs. 6-9). Os mapas de cobalto e ferro sobre eletrodo de platina mostram uma distribuição homogênea desses elementos e os sinais de cobalto e ferro no depósito ficam equivalentes para concentrações de $\mathrm{Fe} \geq 1 \times 10^{-2} \mathrm{~mol} \mathrm{~L}^{-1}$.

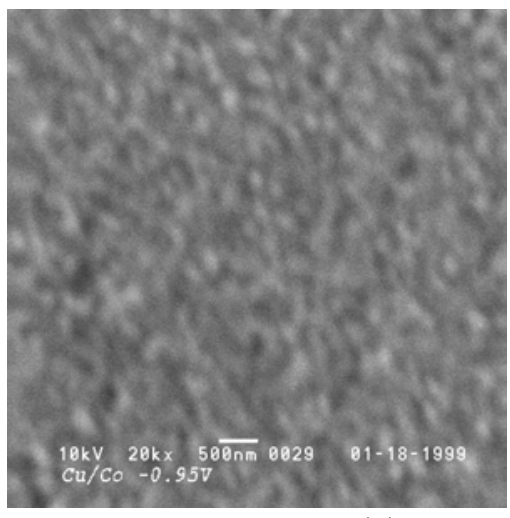

(a)

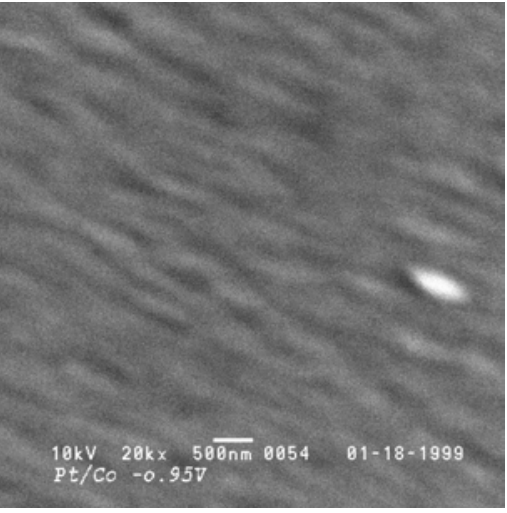

(b)

Figura 6. Micrografias eletrônicas de varredura para o filme de cobalto puro sobre substratos de cobre (a) e platina (b), $E_{d}=-0.95 \mathrm{~V}, \mathrm{t}_{\mathrm{d}}=120 \mathrm{~s}$.

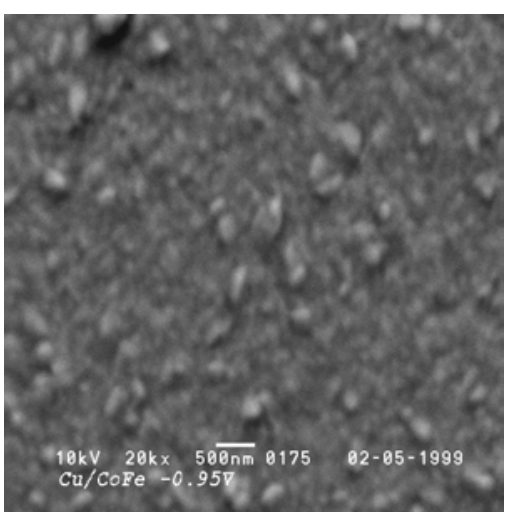

(a)

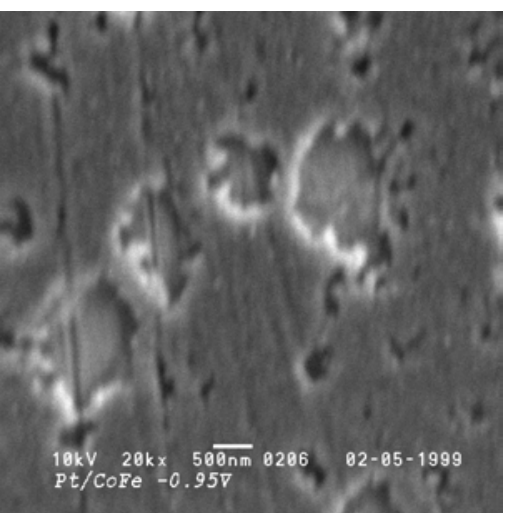

(b)

Figura 7. Micrografias eletrônicas de varredura para o filme de cobalto + ferro $\left(5 \times 10^{-3} \mathrm{~mol} \mathrm{~L}^{-1}\right)$ sobre substratos de cobre (a) e platina (b), $\mathrm{E}_{\mathrm{d}}=-0.95 \mathrm{~V}, \mathrm{t}_{\mathrm{d}}=$ $120 \mathrm{~s}$. 

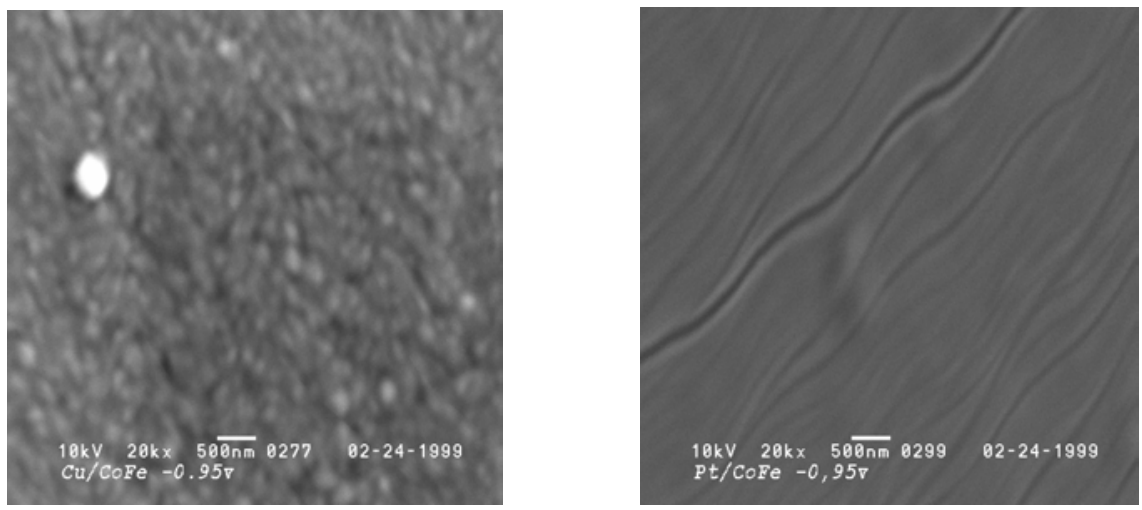

(a)

(b)

Figura 8. Micrografias eletrônicas de varredura para o filme de cobalto + ferro $\left(1 \times 10^{-2} \mathrm{~mol} \mathrm{~L}^{-1}\right)$ sobre substratos de cobre (a) e platina (b), $E_{d}=-0.95 \mathrm{~V}, \mathrm{t}_{\mathrm{d}}=$ $120 \mathrm{~s}$.

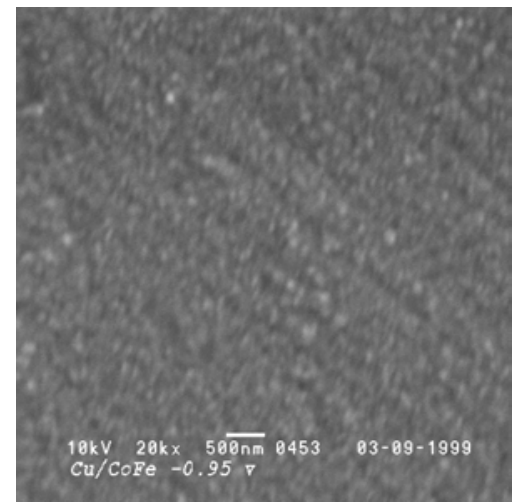

(a)

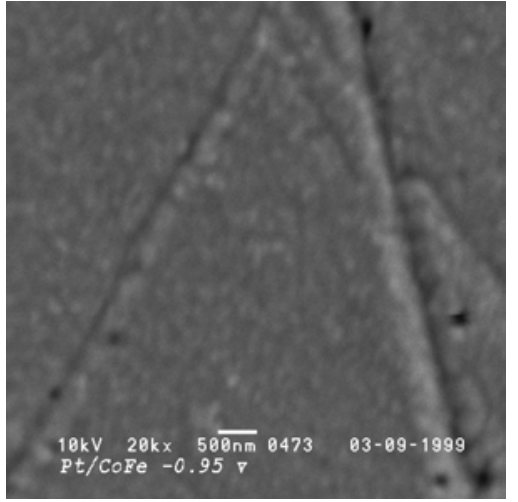

(b)

Figura 9. Micrografias eletrônicas de varredura para o filme de cobalto + ferro $\left(2 \times 10^{-2} \mathrm{~mol} \mathrm{~L}^{-1}\right)$ sobre substratos de cobre (a) e platina (b), $E_{d}=-0.95 \mathrm{~V}, \mathrm{t}_{\mathrm{d}}=$ $120 \mathrm{~s}$.

Os espectros de XPS dos filmes de cobalto puro e de cobalto-ferro, depositados sobre platina e cobre, apresentam valores de energia de ligação para o sinal de Co2p p $_{3 / 2}$ (779.9 $\mathrm{eV})$ correspondentes à um óxido de cobalto, enquanto que o sinal de Fe2 $\mathrm{p}_{3 / 2}(706.6 \mathrm{eV})$ corresponde ao do ferro metálico. O sinal de cobalto metálico, Co2 $\mathrm{p}_{3 / 2}$, aparece em $778.0 \mathrm{eV}$. As Tabelas 1 e 2 mostram os valores de energia de ligação para os filmes de cobalto puro e cobalto-ferro depositados sobre substratos de platina e cobre, respectivamente, $E_{d}=-0.95 \mathrm{~V}, \mathrm{com} \mathrm{t}_{\mathrm{d}}=120 \mathrm{~s}$. A análise da variação das concentrações relativas dos elementos detectados no decorrer das análises de XPS, nos espectros registrados durante a remoção gradativa dos filmes de cobalto puro e cobalto-ferro [ 0.5 , 1 e 2) $\times 10^{-3} \mathrm{~mol} \mathrm{~L}^{-1}$ ], mostrou que para o filme de cobalto-ferro obtidos em solução de $\mathrm{Fe}^{2+} 5 \times 10^{-3} \mathrm{~mol} \mathrm{~L}^{-1}$, o ferro contribuiu menos que 5\% na composição relativa do filme, 
enquanto que cobalto-ferro obtidos em soluções de $\mathrm{Fe}^{2+} 1 \times 10^{-2} \mathrm{~mol} \mathrm{~L}^{-1}$ e $2 \times 10^{-2} \mathrm{~mol}$ $\mathrm{L}^{-1}$ contribuíram em $\sim 5 \%$ e $\sim 10 \%$, respectivamente.

Tabela 1. Valores de energia de ligação dos filmes de cobalto puro e cobaltoferro depositados sobre substrato de platina, $\mathrm{E}_{\mathrm{d}}=-0.95 \mathrm{~V}, \mathrm{t}_{\mathrm{d}}=120 \mathrm{~s}$.

\begin{tabular}{|c|c|c|c|}
\hline Filme & Pt4f (eV) & Co2p3 (eV) & Fe2p3 (eV) \\
\hline $\mathrm{Co}$ & $\begin{array}{l}\left(\mathbf{1}^{\mathbf{0}}\right) 70.79\left(\mathrm{Pt}^{0}\right) \\
\left(\mathbf{1 5}^{\mathbf{0}}\right) 70.64\left(\mathrm{Pt}^{0}\right)\end{array}$ & $\begin{array}{l}777.86\left(\mathrm{Co}^{0}\right) \\
777.75\left(\mathrm{Co}^{0}\right)\end{array}$ & - \\
\hline $\begin{array}{c}\mathrm{CoFe}\left(5 \times 10^{-3}\right. \\
\left.\mathrm{mol} \mathrm{L}^{-1}\right)\end{array}$ & $\begin{array}{l}\left(\mathbf{1}^{\mathbf{0}}\right) 70.85\left(\mathrm{Pt}^{0}\right) \\
\left(\mathbf{1 5}^{\mathbf{0}}\right) 70.70\left(\mathrm{Pt}^{0}\right)\end{array}$ & $\begin{array}{c}780.63\left(\mathrm{CoO}_{\mathrm{x}}\right) \\
777.68\left(\mathrm{Co}^{0}\right)\end{array}$ & $\begin{array}{l}706.92\left(\mathrm{Fe}^{0}\right) \\
707.04\left(\mathrm{Fe}^{0}\right)\end{array}$ \\
\hline $\begin{array}{c}\operatorname{CoFe}\left(1 \times 10^{-2}\right. \\
\left.\mathrm{mol} \mathrm{L}^{-1}\right)\end{array}$ & $\begin{array}{l}\left.\mathbf{1}^{\mathbf{0}}\right) 70.69\left(\mathrm{Pt}^{0}\right) \\
\left(\mathbf{1 5}^{\mathbf{0}}\right) 70.64\left(\mathrm{Pt}^{0}\right)\end{array}$ & $\begin{array}{c}780.92\left(\mathrm{CoO}_{\mathrm{x}}\right) \\
777.70\left(\mathrm{Co}^{0}\right)\end{array}$ & $\begin{array}{l}706.65\left(\mathrm{Fe}^{0}\right) \\
706.40\left(\mathrm{Fe}^{0}\right)\end{array}$ \\
\hline $\begin{array}{c}\mathrm{CoFe}\left(2 \times 10^{-2}\right. \\
\left.\mathrm{mol} \mathrm{L}^{-1}\right)\end{array}$ & 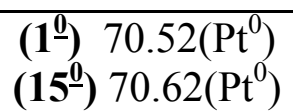 & $\begin{array}{c}780.90\left(\mathrm{CoO}_{\mathrm{x}}\right) \\
777.67\left(\mathrm{Co}^{0}\right)\end{array}$ & $\begin{array}{l}706.30\left(\mathrm{Fe}^{0}\right) \\
706.35\left(\mathrm{Fe}^{0}\right)\end{array}$ \\
\hline
\end{tabular}

Tabela 2. Valores de energia de ligação dos filmes de cobalto puro e cobaltoferro depositados sobre substrato de cobre, $E_{d}=-0.95, t_{d}=120 \mathrm{~s}$.

\begin{tabular}{ccc}
\hline Filme & Co2p3 (eV) & Fe2p3 (eV) \\
\hline $\mathrm{Co}$ & $\begin{array}{c}\left(\mathbf{1}^{\mathbf{0}}\right) 781.70 \mathrm{Co}(\mathrm{OH})_{2} \\
\left(\mathbf{1 2}^{\mathbf{0}}\right) 777.57\left(\mathrm{Co}^{0}\right)\end{array}$ & - \\
\hline $\mathrm{CoFe}\left(5 \times 10^{-3} \mathrm{~mol} \mathrm{~L}^{-1}\right)$ & $\begin{array}{c}\left(\mathbf{1}^{\mathbf{0}}\right) 780.99\left(\mathrm{CoO}_{\mathrm{x}}\right) \\
\left(\mathbf{6}^{\mathbf{0}}\right) 777.66\left(\mathrm{Co}^{0}\right)\end{array}$ & $706.34\left(\mathrm{Fe}^{0}\right)$ \\
\hline $\mathrm{CoFe}\left(1 \times 10^{-2} \mathrm{~mol} \mathrm{~L}^{-1}\right)$ & $\begin{array}{c}\left(\mathbf{1}^{\mathbf{0}}\right) 780.90\left(\mathrm{CoO}_{\mathrm{x}}\right) \\
\left(\mathbf{1 6}^{\mathbf{0}}\right) 777.61\left(\mathrm{Co}^{0}\right)\end{array}$ & $706.58\left(\mathrm{Fe}^{0}\right)$ \\
\hline $\mathrm{CoFe}\left(2 \times 10^{-2} \mathrm{~mol} \mathrm{~L}^{-1}\right)$ & $\left(\mathbf{1}^{\mathbf{0}}\right) 781.01 \mathrm{Co}\left(\mathrm{OH}_{2}\right)_{2}$ & \\
& $\left(\mathbf{1 2}^{\mathbf{0}}\right) 777.68\left(\mathrm{Co}^{0}\right)$ & $706.38\left(\mathrm{Fe}^{0}\right)$ \\
\hline
\end{tabular}

\section{Conclusões}

A eletrodeposição dos filmes de cobalto + ferro foi possível apesar do baixo conteúdo de ferro nas soluções. O ferro deposita-se juntamente com o cobalto, e o teor de ferro no filme cresce com a sua concentração em solução.

Para potenciais de deposição mais negativos que $-0.80 \mathrm{~V}$ o pico agudo de corrente que aparece nos voltamogramas de dissolução pode ser atribuído à dissolução do cobalto; já o pré-pico de oxidação pode estar associado a diferentes estados energéticos de cobalto, fases ou hidretos de cobalto. 
Os resultados dos transientes com o substrato de platina mostraram uma concordância razoável com o modelo de nucleação instantânea, enquanto que com o substrato de cobre ocorreu uma maior concordância para o modelo de nucleação progressiva.

As micrografias eletrônicas de varredura mostraram que os filmes apresentam uma rugosidade maior quando depositados sobre substratos de cobre e as partículas esféricas diminuem com o aumento da concentração de ferro na solução. Os mapas de cobalto e ferro sobre substrato de platina mostram uma distribuição homogênea desses elementos e os sinais de cobalto e ferro no depósito ficam equivalentes para concentrações de $\mathrm{Fe} \geq 1 \times 10^{-2} \mathrm{~mol} \mathrm{~L}^{-1}$.

Os valores de energia de ligação dos filmes de cobalto puro e de cobalto-ferro depositados sobre platina $\left(E_{d}=-0.95 \mathrm{~V}, \mathrm{t}_{\mathrm{d}}=120 \mathrm{~s}\right)$ e cobre $\left(\mathrm{E}_{\mathrm{d}}=-1.10 \mathrm{~V}, \mathrm{t}_{\mathrm{d}}=\right.$ $120 \mathrm{~s}$ ) apresentam, para o primeiro ciclo, os valores de energia de ligação para o sinal de $\mathrm{Co} 2 \mathrm{p}_{3 / 2}$ correspondentes à um óxido de cobalto, enquanto que o sinal de $\mathrm{Fe} 2 \mathrm{p}_{3 / 2}$ corresponde ao do ferro metálico. O sinal de cobalto metálico, Co2 $\mathrm{p}_{3 / 2}$, aparece em $778.0 \mathrm{eV}$.

\section{Agradecimentos}

Os autores agradecem ao Conselho Nacional de Desenvolvimento Científico e Tecnológico $(\mathrm{CNPq})$ pelas bolsas recebidas e ao Servèis Científico Tècnics da Universitat de Barcelona pelas facilidades no uso da Unitat TEMIXPS.

\section{Referências}

1. J.M. Baibich, A. Fert Broto, F.N. Van Dau, F. Petroff, P. Etienne, G. Creuzet, A. Friederich, Chazelas, J. Phys. Rev. Lett. 61 (1988) 2472.

2. E. Chassaing, P. Nallet, M.F. Trichet, J. Electrochem. Soc. 143 (1995) L98.

3. J.A. Switzer, M.G. Shumsky, E.W. Bohannam, Science 284 (1999) 293.

4. S. Kashiwabara, Y. Jyoko, Y. Hayashi, Mater. Trans. 37 (1996) 289.

5. A. Barthelemy, V. Cros, J.L. Duvail, A. Fert, R. Morel, F. Parent, F. Petroff, L.B. Steren, Nano. Mat. 6 (1995) 217.

6. P.M. Levy, Science 264 (1994) 413. 
7. H.F. Quinn, I.M. Croll, Advances in X-Ray Analysis. New York: Plenum Press, Vol. 4, (1980).

8. E. Gomez, M. Marin, F. Sanz, E. Valles, J. Electroanal. Chem. 422 (1997) 139.

9. P. Cook, Advances Materials \& Processes 4 (1994) 27.

10. C.C. Yang, H.Y. Cheh, J. Electrochem. Soc. 142 (1995) 755.

11. D.R. Lashmore, R. Thomson, J. Mater. Res. 7 (1992) 2379.

12. J.F. Moulder, W.F. Stickle, P.E. Sobol, K.D. Bomben, Handbook of X-Ray Photoelectron Spectroscopy, Minnesota: Jill Chastain (1992).

13. E. Soto, M. Arce, M. Palomar-Pardavé, I. González, Electrochim. Acta 41 (1996) 2647.

14. E.M. Kakuno, D.H. Mosca, I. Mazzaro, N. Mattoso, W.H. Schreiner, M.A.B. Gomes, J. Eletrochem. Soc. 144 (1997) 3222.

15. V.M. López-Hirata, E.M. Arce-Estrada, Electrochim. Acta 42 (1997) 61.

16. I. Kirilova, I. Ivanov, St. Rashkov, J. App. Electrochem. 28 (1998) 637.

17. R. Bertazzoli, D. Pletcher, Electrochim. Acta 38 (1993) 671.

18. G. Guanawardena, G. Hills, I. Montenegro, J. Electroanal. Chem. 138 (1982) 241.

19. B. Scharifker, G. Hills, Electrochim. Acta 28 (1983) 879.

20. B. Scharifker, J. Mostany, J. Electroanal. Chem. 177 (1984) 13. 